\title{
Self-Perceived Quality of Life Among Patients with Alzheimer's Disease: Two Longitudinal Models of Analysis
}

\author{
Josep L. Conde-Sala ${ }^{\mathrm{a}, \mathrm{b}, *}$, Oriol Turró-Garriga ${ }^{\mathrm{b}}$, Cristina Portellano-Ortiz $^{\mathrm{a}}$, Vanesa Viñas-Diez ${ }^{\mathrm{c}}$, \\ Jordi Gascón-Bayarric and Ramón Reñé-Ramírez ${ }^{\mathrm{c}}$ \\ ${ }^{a}$ Faculty of Psychology, University of Barcelona, Spain \\ ${ }^{\mathrm{b}}$ Aging, Disability and Health Research Group, Biomedical Research Institute of Girona (IdIBGi), \\ Catalonia, Spain \\ ${ }^{\mathrm{c}}$ Department of Neurology, Dementia Unit, Bellvitge University Hospital, Hospitalet de Llobregat, Spain
}

Handling Associate Editor: Marcia Cristina Nascimento Dourado

Accepted 22 February 2016

\begin{abstract}
The objective was to analyze the factors that influence self-perceived quality of life (QoL) in patients with Alzheimer's disease (AD), contrasting two different longitudinal models. A total of 127 patients were followed up over 24 months. The instruments applied were: Quality of Life in Alzheimer's Disease scale (QoL-AD), Geriatric Depression Scale-15, Anosognosia Questionnaire-Dementia, Disability Assessment in Dementia, Neuropsychiatric Inventory, and the Mini-Mental State Examination. Two models for grouping patients were tested: 1) Baseline score on the QoL-AD (QoLBaseline), and 2) Difference in QoL-AD score between baseline and follow-up (QoL-Change). Generalized estimating equations were used to analyze longitudinal data, and multinomial regression analyses were performed. Over the follow-up period the QoL-Baseline model showed greater variability between groups (Wald $\chi^{2}=172.3, p<0.001$ ) than did the QoLChange model (Wald $\chi^{2}=1.7, p=0.427$ ). In the QoL-Baseline model the predictive factors were greater depression (odds ratio $[\mathrm{OR}]=1.20$; $95 \% \mathrm{CI}: 1.00-1.45)$ and lower functional ability $(\mathrm{OR}=0.92 ; 95 \% \mathrm{CI}: 0.85-0.99)$ for the Low QoL group ( $<33$ QoL-AD), and less depression $(\mathrm{OR}=0.68$; 95\% CI: 0.52-0.88), more anosognosia (OR $=1.07$; 95\% CI: $1.01-1.13)$, and fewer neuropsychiatric symptoms ( $\mathrm{OR}=0.95 ; 95 \%$ CI: 0.91-0.99) for the High-QoL group ( $>37 \mathrm{QoL}-\mathrm{AD})$. The model based on baseline scores (QoL-Baseline) was better than the QoL-Change model in terms of identifying trajectories and predictors of QoL in AD.
\end{abstract}

Keywords: Alzheimer's disease, analytic models, anosognosia, depression, longitudinal study, quality of life

\section{INTRODUCTION}

In its report, Dementia: A Public Health Priority, the World Health Organization [1] highlights the

\footnotetext{
${ }^{*}$ Correspondence to: Josep L. Conde-Sala, Faculty of Psychology, University of Barcelona, Passeig Vall d'Hebron, 171, 08035 Barcelona, Spain. Tel.: +34 93 3125814; Fax: +34 93 4021368; E-mail: jllconde@ub.edu.
}

need to develop programs that can improve the social wellbeing and quality of life $(\mathrm{QoL})$ of people with dementia. Consequently, it is important to identify the factors that influence QoL so as to design interventions that might improve it.

Among research that has examined predictors of change in the QoL of patients with dementia, longitudinal studies are of particular interest. The majority of these studies have found, when considering samples 
as a whole, that mean QoL scores remain relatively stable over time [2-14], although some research conducted in residential settings has reported a decrease [15-17].

The factors associated with perceived QoL are consistent across some studies. Greater depression appears to be the most relevant aspect and it is associated with a deteriorating QoL over time [2, 6-8, 18]. A greater number of neuropsychiatric symptoms (higher scores on the NPI) is also associated with poorer perceived QoL [5, 11, 17], whereas better functional ability correlates with higher QoL scores $[3,6]$ Cognitive changes appear to have only a weak or no effect $[12,19]$.

Many longitudinal studies also distinguish subgroups of patients according to whether their perceived QoL deteriorates, remains stable, or improves over time. The combined data of five community studies $[3,7-9,13]$, with a total sample of 417 people, indicate that QoL scores deteriorated in $28.2 \%$, remained stable in $35.9 \%$, and improved in $35.7 \%$. Across six studies carried out in residential care settings [2, 4, 14-17], with a total sample of 906 elders, QoL scores deteriorated in $41 \%$, remained stable in $27.2 \%$, and improved in $31.7 \%$.

Analyzing the self-perceived QoL of patients is, however, a complex task [15] due to the considerable individual variability and small effect sizes involved. It should also be noted that the severity of dementia does not seem to affect QoL scores [12, 13]. Baseline QoL appears to have a predictive value, although studies report contrasting results as regards the direction of the effect: While some studies have found that higher QoL at baseline was associated with better QoL at follow-up [2, 6, 7, 9, 15, 16], others, mostly in residential settings, report a reduction in perceived QoL among those with higher baseline QoL scores $[4,14,17]$.

Despite this evidence, most studies aimed at analyzing the evolution of $\mathrm{QoL}$ have used the total QoL score or have defined groups based on how their scores changed (QoL-Change: Deterioration/stability/improvement). However, given the predictive capacity of baseline QoL and the stability of scores over time, it might be more useful to define groups on the basis of their baseline score (Low, Medium, and High QoL), to analyze how these scores change, and to identify predictive factors for each group at the time of the baseline assessment. This distribution of groups could then be compared with that used by the majority of studies (i.e., QoLChange: Decreased, No change, Increased).
The hypothesis underlying the present study is that a model in which groups are defined according to their baseline QoL score would be better at identifying the trajectories and predictive factors of QoL, and that these factors (e.g., depression, neuropsychiatric symptoms, anosognosia, and functional ability) would not be the same for each group.

Given the wide range of factors that are potentially involved and the various models that have been used to study changes in the perceived QoL of patients with dementia, the present study had the following objectives: 1) to analyze the factors that influence self-perceived QoL when the total sample of patients is considered; 2) to analyze the factors associated with self-perceived QoL when three subgroups are established on the basis of the baseline QoL score (QoLBaseline model) and according to the change in score over time (QoL-Change model); and 3) to assess which of these models is best in terms of identifying the trajectory and predictors of QoL scores over time.

\section{METHODS}

\section{Design and study population}

This was a longitudinal study involving a 24-month follow-up of a consecutive sample of outpatients seen at the Dementia Unit (Department of Neurology) of Bellvitge University Hospital (Hospitalet de Llobregat, Barcelona). They were all diagnosed as either AD according to the criteria of the Diagnostic and Statistical Manual of Mental Disorders (DSM-5) [20] or probable $\mathrm{AD}$ according to the criteria of the National Institute of Neurological and Communicative Disorders and Stroke / Alzheimer's Disease and Related Disorders Associations [21]. In addition, they all scored between 10 and 28 on the Mini-Mental State Examination (MMSE) [22], thereby enabling a quality-of-life scale (QoL-AD) [23] to be administered. The main caregiver was defined as the person with ongoing responsibility for helping the patient with activities of daily living (ADL). All the caregivers were relatives of the patients.

Patients were excluded if they presented vascular or traumatic events, alcohol or substance dependence or abuse, and if they had severe communication problems that prevented them from responding adequately to the assessment instruments. Informed consent was obtained for all participants. The study was approved by the hospital's Clinical Research Ethics Committee (ref. PR162/10). 


\section{Instruments}

Socio-demographic data for patients were gathered using a structured questionnaire designed by the researchers.

- Quality of life. The Quality of Life in Alzheimer's Disease (QoL-AD) scale [23] was used to explore patients' own views regarding their quality of life. This instrument comprises 13 items that refer to different aspects of the patient's wellbeing. Each item is scored on a Likert-type scale ranging from 1 (poor) to 4 (excellent), yielding a total score between 13 and 52 (the higher the score the better the perceived quality of life).

- Behavioral and psychological symptoms of dementia. This aspect was evaluated by means of the Neuropsychiatric Inventory (NPI) [24], which comprises 12 subscales that assess the frequency and severity of 12 neuropsychiatric symptoms, based on information provided by caregivers. Scores range from 0 to 144 , and the higher the score the greater the frequency and severity of neuropsychiatric symptoms.

- Anosognosia. The Anosognosia QuestionnaireDementia (AQ-D) [25] was administered to patients and caregivers. It comprises 30 items referring to cognitive/functional deficits and changes in the patient's behavior, with each item being rated according to the frequency of occurrence, from 0 (never) to 3 (always). The total score therefore ranges from 0 to 90 . The degree of anosognosia is estimated on the basis of the difference between patient and caregiver scores; the greater the difference the greater the anosognosia.

- Cognitive assessment of the patient. This was based on the MMSE [22], a brief cognitive assessment tool whose score ranges from 0 to 30 (the lower the score the greater the cognitive deterioration). Patients' scores were corrected for age and level of education [26].

- Depression in the patient. This was assessed using the Geriatric Depression Scale (GDS), in its 15-item format [27]. The cut-off score for probable depression is 6 , while a score of 10 or higher is indicative of depression. The 15 -item version of the GDS has been previously used to assess depression in patients with $\mathrm{AD}[28,29]$.

- Functional assessment of the patient. The Disability Assessment for Dementia (DAD) [30] is a measure of basic and instrumental ADL and it was administered to the main caregiver. The DAD comprises 40 items and its total score ranges from 40 to 80 (the higher the score the greater the patient's functional ability).

- Severity of dementia. This was based on the criteria of the Global Deterioration Scale (GDS) [31], a tool designed to determine the stage of a patient's dementia. Patients were excluded if they were classified as either GDS 7, due to the severity of their dementia, or GDS 3, due to the possible confusion with mild cognitive impairment.

\section{Procedure}

Neurologists from the Dementia Unit of Bellvitge University Hospital selected eligible patients according to the inclusion criteria and then determined their stage of dementia using the GDS. The initial sample was recruited between January and December 2011, with a 24-month follow-up assessment being conducted in May 2014. The baseline sample comprised 221 patients, of whom 127 were able to be re-assessed at 24 months. Regarding the $94(42.5 \%)$ patients lost to follow-up, 27 (28.7\%) had entered residential care, $31(32.9 \%)$ decided not to participate any further, $2(2.1 \%)$ had changed address, and 34 (36.1\%) had died. The present study only uses data from the 127 participants who completed the 24-month follow-up.

The aims of the study were explained to all participants prior to the baseline interview, and informed consent was obtained from both patients and caregivers before proceeding. Patients and their caregivers were then interviewed separately by two clinical psychologists trained in the administration of the respective tests.

\section{Models of longitudinal analysis}

In order to study the factors associated with changes in perceived QoL, we designed two models for grouping the scores obtained by patients on the QoL-AD. The first was based on their score at baseline (QoL-Baseline) and yielded three groups that were comparable in terms of the number of subjects in each: Low-QoL $(<33, n=40)$, Medium-QoL (33-37, $n=43)$, and High-QoL (>37, $n=44)$. The second model was based on the difference between QoL-AD scores at baseline and 24-month follow-up (QoL-Change), and here we established three groups 
based on the cut-off points that have generally been used in previous studies $[2,4,9,14]$ : Decreased QoL $(\leq 3, n=25)$, No change $( \pm 2, n=44)$, and Increased QoL $(\geq 3, n=58)$. The number of groups was the same at all the assessment points and in both models.

\section{Statistical analysis}

For the longitudinal analysis of data we applied generalized estimating equations (GEE) and the Wald $\chi^{2}$ statistic [32], in conjunction with a first-order autoregressive working correlation matrix (AR1). This approach enabled us to examine the general effects of the independent variables on the dependent variable (QoL-AD), with respect to the factors time, patient group, and the interaction between the two (Time* Group), as well as the simple effects of differences between the groups and the assessment points.

The effect size of the difference between several means was assessed using eta squared $\left(\eta^{2}\right)$, the value of which was interpreted according to the following criteria: $<0.06$, weak effect; $0.06-0.13$, moderate effect; $>0.13$, strong effect [33].

A multivariate analysis was first performed for the whole sample, taking the QoL-AD score as the dependent variable and patient factors (introduced simultaneously) as independent variables, and specifying the parameter estimates at each of the three measurement points (baseline, 12 months, and 24 months).

In order to detect the factors that predicted patients' group assignment in the two models we performed multinomial regression analyses at baseline, using the 'Main effects' option.

For hypothesis contrasts the level of statistical significance was set at 0.05 . All data processing and analysis was performed using SPSS, v22.0 para Windows (SPSS Inc.; Chicago).

\section{RESULTS}

Comparison of the study sample with the baseline sample lost to follow-up

The study sample comprised the 127 patients and caregivers who, from among the 221 initially recruited, completed the full 24-month followup. At baseline, the cases lost to follow-up $(n=94)$ were older than those in the study sample (79.4 \pm 6.9 versus $76.6 \pm 7.3 ; z=3.0, p=0.002)$, and they were also more impaired, presenting a poorer cognitive status $(\mathrm{MMSE}=17.2 \pm 5.5$ versus $19.1 \pm 5.2 ; z=2.4, p=0.014$ ), lower functional ability $(\mathrm{DAD}=54.7 \pm 9.5$ versus $60.6 \pm 10.0 ; z=4.2$, $p<0.001$ ), greater anosognosia (AQ-D $=38.9 \pm 18.2$ versus $30.2 \pm 18.2 ; t=3.4, p=0.001)$, and more neuropsychiatric symptoms $(\mathrm{NPI}=31.2 \pm 21.9$ versus $20.9 \pm 16.4 ; z=3.9, p<0.001)$.

Self-perceived QoL at baseline did not differ significantly between lost cases and those who were followed up (QoL-AD $=35.2 \pm 4.9$ versus $34.9 \pm 4.7 ; z=0.8, p=0.402)$. Neither did the QoL$\mathrm{AD}$ scores of lost cases vary according to the severity of dementia (GDS stage, $\chi^{2}=4.0, p=0.131$ ).

\section{Sociodemographic and clinical characteristics of the study sample}

At baseline, patients had a mean age of $76.6 \pm 7.3$ years, 82 of them $(64.6 \%)$ were women, and 43 $(33.9 \%)$ had received five or more years of formal education.

Across the follow-up period we observed a moderate deterioration in cognitive status (MMSE, $\eta^{2}=0.10$ ), a notable decrease in functional ability (DAD, $\eta^{2}=0.16$ ), and an increased presence of both neuropsychiatric symptoms (NPI, $\left.\eta^{2}=0.03\right)$ and anosognosia (AQ-D, $\eta^{2}=0.02$ ), with a small effect size. Patients' ratings of their own QoL did not change significantly during follow-up $(p=0.247)$. Complete data shown in Table 1.

\section{Factors affecting QoL ratings during follow-up in the whole sample}

The multivariate analysis (Table 2) showed that depression had an important negative effect on selfperceived QoL at all three assessment points. Greater anosognosia had a positive effect on QoL-AD scores that likewise continued across the follow-up period. The positive effect of greater functional ability was only significant at 12 and 24 months. Neuropsychiatric symptoms and cognitive status had no significant effect on QoL scores.

The severity of dementia (assessed by GDS stage) had little effect on QoL-AD scores, and the differences between severity groups (GDS stages 4, 5 , and 6) were not significant at baseline (Wald $\chi^{2}=1.0, p=0.585$ ), at 12 months (Wald $\chi^{2}=0.8$, $p=0.661$ ), or at 24 months (Wald $\chi^{2}=0.3, p=0.836$ ). With respect to the general effects of the model, only Time was significant (Wald $\chi^{2}=6.4, p=0.040$ ), there being a slight increase in QoL-AD scores at 
Table 1

Sociodemographic and clinical data of patients

\begin{tabular}{|c|c|c|c|c|c|c|c|}
\hline & \multirow{2}{*}{$\begin{array}{c}\text { Baseline } \\
1(n=127)\end{array}$} & \multirow{2}{*}{$\begin{array}{c}12 \text { months } \\
2(n=127)\end{array}$} & \multirow{2}{*}{$\frac{24 \text { months }}{3(n=127)}$} & \multicolumn{4}{|c|}{ Differences } \\
\hline & & & & Test & $d f$ & $p$ & $\eta^{2}$ \\
\hline Age, mean (SD) & $76.6(7.3)$ & $77.8(7.2)$ & $79.0(7.2)^{b}$ & $7.1^{1}$ & 2 & 0.025 & \\
\hline Women, $n(\%)$ & $82(64.6)$ & $82(64.6)$ & $82(64.6)$ & $0.0^{2}$ & 2 & 1.000 & \\
\hline Marital status (widowed), $n(\%)$ & $37(30.1)$ & $38(30.9)$ & $40(32.0)$ & $0.1^{2}$ & 2 & 0.948 & \\
\hline Schooling (> 5 years), $n(\%)$ & $43(33.9)$ & $43(33.9)$ & $43(33.9)$ & $0.0^{2}$ & 2 & 1.000 & \\
\hline Cognition (MMSE), mean (SE) & $19.1(0.4)$ & $16.8(0.5)^{\mathrm{a}}$ & $13.9(0.6)^{\mathrm{b}, \mathrm{c}}$ & $106.1^{3}$ & 2 & $<0.001$ & 0.10 \\
\hline Function (DAD), mean (SE) & $60.6(0.8)$ & $55.5(0.8)^{\mathrm{a}}$ & $50.0(0.8)^{\mathrm{b}, \mathrm{c}}$ & $201.4^{3}$ & 2 & $<0.001$ & 0.16 \\
\hline Behavior (NPI), mean (SE) & $20.9(1.4)$ & $23.4(1.4)$ & $28.6(1.5)^{\mathrm{b}, \mathrm{c}}$ & $25.5^{3}$ & 2 & $<0.001$ & 0.03 \\
\hline Anosognosia (AQ-D), mean (SE) & $30.2(1.6)$ & $34.5(1.7)$ & $37.9(1.6)^{\mathrm{b}}$ & $44.4^{3}$ & 2 & $<0.001$ & 0.02 \\
\hline Depression (GDS), mean (SE) & $3.3(0.2)$ & $3.4(0.2)$ & $3.1(0.2)$ & $3.9^{3}$ & 2 & 0.137 & 0.00 \\
\hline Quality of life (QoL-AD), mean (SE) & $34.9(0.4)$ & $35.1(0.4)$ & $35.9(0.4)^{\mathrm{b}, \mathrm{c}}$ & $16.9^{3}$ & 2 & $<0.001$ & 0.000 \\
\hline \multicolumn{8}{|l|}{ GDS stage, $n(\%)$} \\
\hline GDS 4 & $68(53.5)$ & $33(26.0)$ & $12(9.4)$ & $83.6^{2}$ & 4 & $<0.001$ & \\
\hline GDS 5 & $43(33.9)$ & $52(40.9)$ & $40(31.5)$ & $\mathrm{V}=0.33$ & & & \\
\hline GDS 6 & $16(12.6)$ & $42(33.1)$ & $75(59.1)$ & & & & \\
\hline \multicolumn{8}{|l|}{ Family caregivers, $n(\%)$} \\
\hline Spouse & $72(56.7)$ & $72(56.7)$ & $70(55.2)$ & $0.2^{2}$ & 4 & 0.992 & \\
\hline Adult-child & $51(40.2)$ & $52(40.9)$ & $53(41.7)$ & $\mathrm{V}=0.01$ & & & \\
\hline Other & $4(3.1)$ & $3(2.4)$ & $4(3.1)$ & & & & \\
\hline \multicolumn{8}{|l|}{ Living with caregiver, $n(\%)$} \\
\hline Yes & $101(79.5)$ & $100(78.7)$ & $97(76.4)$ & $0.4^{2}$ & 2 & 0.819 & \\
\hline No & $26(20.5)$ & $27(21.3)$ & $30(23.6)$ & $V=0.03$ & & & \\
\hline
\end{tabular}

${ }^{1}$ Kruskal-Wallis; ${ }^{2}$ Chi square test for categorical variables; V, Cramer's V; ${ }^{3}$ Generalized estimating equations, Wald $\chi^{2}$, for time. Means, estimated marginal; SE, standard error; Significant with Bonferroni post hoc contrasts: ${ }^{\mathrm{a}} 1-2,{ }^{\mathrm{b}} 1-3,{ }^{\mathrm{c}} 2-3 ; \eta^{2}=$ Eta-squared (effect size); $p$-values $<0.05$ are shown in bold. MMSE, Mini-Mental State Examination; DAD, Disability Assessment in Dementia; NPI, Neuropsychiatric Inventory; AQ-D, Anosognosia Questionnaire-Dementia; GDS, Geriatric Depression Scale; GDS stage, Global Deterioration Scale; QoL-AD, Quality of Life in Alzheimer's Disease scale.

Table 2

Multivariate analysis. QoL-AD, Time \& Factors, in the overall sample

\begin{tabular}{|c|c|c|c|c|c|c|c|c|c|c|c|c|}
\hline & \multicolumn{12}{|c|}{ Parameter estimates } \\
\hline & \multicolumn{3}{|c|}{ Model effects } & \multicolumn{3}{|c|}{ Baseline } & \multicolumn{3}{|c|}{12 months } & \multicolumn{3}{|c|}{24 months } \\
\hline & $\chi^{2}$ & $d f$ & $p$ & $\mathrm{~B}(\mathrm{SE})$ & $\chi^{2}$ & $p$ & $\mathrm{~B}(\mathrm{SE})$ & $\chi^{2}$ & $p$ & $\mathrm{~B}(\mathrm{SE})$ & $\chi^{2}$ & $p$ \\
\hline \multicolumn{13}{|l|}{ Time* } \\
\hline Depression (GDS) & 71.8 & 3 & $<0.001$ & $-0.66(0.10)$ & 43.1 & $<0.001$ & $-0.72(0.11)$ & 42.8 & $<0.001$ & $-0.80(0.11)$ & 53.2 & $<0.001$ \\
\hline Anosognosia (AQ-D) & 43.0 & 3 & $<0.001$ & $0.07(0.01)$ & 26.7 & $<0.001$ & $0.07(0.01)$ & 26.3 & $<0.001$ & $0.09(0.01)$ & 37.6 & $<0.001$ \\
\hline Function (DAD) & 8.0 & 3 & 0.044 & $0.02(0.02)$ & 1.3 & 0.247 & $0.06(0.02)$ & 5.8 & 0.015 & $0.06(0.02)$ & 4.7 & 0.029 \\
\hline Behavior (NPI) & 5.6 & 3 & 0.130 & $-0.04(0.02)$ & 3.7 & 0.054 & $-0.02(0.01)$ & 3.3 & 0.068 & $-0.01(0.00)$ & 1.2 & 0.262 \\
\hline Cognition (MMSE) & 5.8 & 3 & 0.120 & $0.09(0.05)$ & 2.7 & 0.095 & $-0.02(0.04)$ & 0.2 & 0.618 & $0.00(0.03)$ & 0.0 & 0.822 \\
\hline
\end{tabular}

Method: Dependent variable $=$ QoL-AD; Independent variables $=$ introduced simultaneously. Generalized estimating equations, Wald $\chi^{2} ; \mathrm{B}$, beta coefficient; SE, standard error; $p$-values $<0.05$ are shown in bold. MMSE, Mini-Mental State Examination; DAD, Disability Assessment in Dementia; NPI, Neuropsychiatric Inventory; AQ-D, Anosognosia Questionnaire-Dementia; GDS, Geriatric Depression Scale; QoL-AD, Quality of Life in Alzheimer's Disease scale.

24 months among patients in the GDS 6 group $\left(\eta^{2}=0.007\right)$. Neither the between-groups difference (Wald $\chi^{2}=1.1, p=0.553$ ) or the Time*Group interaction (Wald $\chi^{2}=0.6, p=0.960$ ) were significant.

Regarding sociodemographic variables, men scored slightly higher than women in the model (Wald $\chi^{2}=3.9, p=0.046$ ), although the differences were only significant at 24 months. Participants with $>5$ years of schooling also scored higher (Wald $\chi^{2}=6.4, p=0.011$ ), with differences being significant at 12 and 24 months. In both cases the effect sizes were small $(d<0.5)$. Age, relationship to the caregiver (spouse/adult child), and living with the caregiver were associated with no significant differences between the groups, although scores did increase slightly over the follow-up period for younger participants $\left(\eta^{2}=0.00\right)$, those who lived with the caregiver $\left(\eta^{2}=0.01\right)$, and those who were cared for by a spouse $\left(\eta^{2}=0.00\right)$, the corresponding effect sizes being very small. 
Table 3

Quality of life scores (QoL-AD) for the groups created in the two models

\begin{tabular}{|c|c|c|c|c|c|c|}
\hline & \multirow{2}{*}{$\begin{array}{l}\text { 1. Baseline } \\
\text { Mean (SE) }\end{array}$} & \multirow{2}{*}{$\begin{array}{c}2.12 \text { months } \\
\text { Mean (SE) }\end{array}$} & \multirow{2}{*}{$\begin{array}{c}3.24 \text { months } \\
\text { Mean (SE) }\end{array}$} & \multicolumn{3}{|c|}{ Simple effects } \\
\hline & & & & $\chi^{2}$ & $d f$ & $p$ \\
\hline \multicolumn{7}{|l|}{ A. Groups QoL-Baseline } \\
\hline A1. Low-QoL & $29.4(0.4)$ & $30.9(0.7)^{\mathrm{a}}$ & $31.7(0.7)^{\mathrm{b}}$ & 21.1 & 2 & $<0.001$ \\
\hline A2. Medium & $35.3(0.2)^{\mathrm{d}}$ & $35.5(0.4)^{\mathrm{d}}$ & $36.3(0.4)^{\mathrm{d}}$ & 5.5 & 2 & 0.064 \\
\hline A3 High-QoL & $39.6(0.3)^{\mathrm{e}, \mathrm{f}}$ & $38.6(0.3)^{\mathrm{a}, \mathrm{e}, \mathrm{f}}$ & $39.3(0.4)^{\mathrm{e}, \mathrm{f}}$ & 9.5 & 2 & 0.008 \\
\hline Simple effects $\chi^{2}(d f) p$ & $341.8(2)<\mathbf{0 . 0 0 1}$ & $95.2(2)<\mathbf{0 . 0 0 1}$ & $79.0(2)<\mathbf{0 . 0 0 1}$ & & & \\
\hline - Model $\chi^{2}(d f) p$ & \multicolumn{6}{|c|}{ Time: $18.1(2)<\mathbf{0 . 0 0 1}$; Groups: $172.3(2)<\mathbf{0 . 0 0 1}$; Time* groups: 18.0 (4) $\mathbf{0 . 0 0 1}$} \\
\hline \multicolumn{7}{|l|}{ B. Groups QoL-Change } \\
\hline B1. Decreased-QoL & $37.5(1.0)$ & $34.1(1.1)^{\mathrm{a}}$ & $33.5(1.2)^{\mathrm{b}}$ & 65.8 & 2 & $<0.001$ \\
\hline B2. No change & $34.8(0.6)$ & $34.7(0.7)$ & $35.0(0.6)$ & 4.8 & 2 & 0.090 \\
\hline B3. Increased-QoL & $34.0(0.5)^{\mathrm{e}}$ & $35.9(0.4)^{\mathrm{a}}$ & $37.6(0.5)^{\mathrm{b}, \mathrm{c}, \mathrm{e}, \mathrm{f}}$ & 283.7 & 2 & $<\mathbf{0 . 0 0 1}$ \\
\hline Simple effects $\chi^{2}(d f) p$ & $8.1(2) 0.017$ & $3.4(2) 0.182$ & $15.9(2)<0.001$ & & & \\
\hline - Model $\chi^{2}(d f) p$ & \multicolumn{6}{|c|}{ Time: 5.9 (2) 0.051 ; Groups: 1.7 (2) 0.427; Time* groups: 300.4 (4) $<\mathbf{0 . 0 0 1}$} \\
\hline
\end{tabular}

Generalized estimating equations, Wald $\chi^{2}$; Means, estimated marginal; SE, standard error; Significant with Bonferroni post hoc contrasts: ${ }^{\mathrm{a}} 1-2,{ }^{\mathrm{b}} 1-3,{ }^{\mathrm{c}} 2-3,{ }^{\mathrm{d}} \mathrm{A} 1 / \mathrm{B} 1-\mathrm{A} 2 / \mathrm{B} 2,{ }^{\mathrm{e}} \mathrm{A} 1 / \mathrm{B} 1-\mathrm{A} 3 / \mathrm{B} 3,{ }^{\mathrm{f}} \mathrm{A} 2 / \mathrm{B} 2-\mathrm{A} 3 / \mathrm{B} 3 . p$-values < 0.05 are shown in bold. QoL-AD, Quality of Life in Alzheimer's Disease scale; AQ-D, Anosognosia Questionnaire-Dementia; GDS, Geriatric Depression Scale; NPI, Neuropsychiatric Inventory; DAD, Disability Assessment in Dementia; MMSE, Mini-Mental State Examination.

\section{Variability in baseline QoL-AD scores and clinical factors}

In the QoL-Baseline model the baseline scores on the QoL-AD were as follows: Low $=29.4 \pm 2.7$; Medium $=35.3 \pm 1.4 ; \mathrm{High}=39.6 \pm 2.3\left(\chi^{2}=112.6\right.$, $\left.p<0.001 ; \eta^{2}=0.78\right)$. In the QoL-Change model the baseline scores were: Decreased $=37.5 \pm 5.5$; No change $=34.8 \pm 4.6 ;$ Increased $=34.0 \pm 4.0\left(\chi^{2}=\right.$ 9.4, $\left.p=0.009 ; \eta^{2}=0.07\right)$. Although the differences between groups were significant in both models, the between-group variability was greater in the QoLBaseline model, with a large effect size. Conversely, the within-group variability was lower in the QoLBaseline model, with smaller standard deviations.

At baseline the clinical factors that were significantly associated with between-group variability in the QoL-Baseline model were depression (GDS): Low $=5.2 \pm 3.0$, Medium $=3.3 \pm 2.4$, High $=1.6 \pm$ $1.6\left(\chi^{2}=34.1, p<0.001 ; \eta^{2}=0.26\right)$; functional ability (DAD): Low $=57.5 \pm 9.3$, Medium $=62.5 \pm 10.3$, High $=61.5 \pm 9.8 \quad\left(\chi^{2}=6.5, p=0.038 ; \eta^{2}=0.04\right)$; and neuropsychiatric symptoms (NPI): Low $=24.3$ \pm 17.4 , Medium $=21.3 \pm 15.7, \quad$ High $=17.5 \pm 15.8$ $\left(\chi^{2}=6.3, p=0.043 ; \eta^{2}=0.02\right)$. In the QoL-Change model none of the clinical factors was associated with significant variability between the groups.

In order to examine the predictive ability of the baseline QoL-AD score on QoL scores at 24 months we conducted a linear regression analysis. The results showed that the baseline QoL score was a good predictor of QoL-AD scores at 24 months $\left(\mathrm{R}^{2}=0.588\right.$, $\beta=0.76, t=13.3, p<0.001$.

\section{Changes over time in QoL-AD scores in the two models of analysis}

For the groups created in the QoL-Baseline model the GEE indicated that the general effects were significant for Time, Group, and for the Time*Group interaction. Regarding the simple effects, there were important between-group differences at all three assessment points $\left(\eta^{2} ; \mathrm{T} 1=0.78, \mathrm{~T} 2=0.45\right.$; $\mathrm{T} 3=0.41$ ), with higher scores being observed in the High-QoL group. During follow-up, scores in the Low-QoL group increased, whereas those in the High-QoL group decreased, the effect size being weak in both cases $\left(\eta^{2} ;\right.$ Low $=0.04$, High $\left.=0.02\right)$.

For the groups created in the QoL-Change model the GEE indicated that the general effects were only significant for the Time*Group interaction. Regarding the simple effects, a between-groups difference of moderate magnitude was observed at baseline $\left(\eta^{2}=0.07\right)$ and at 24 months $\left(\eta^{2}=0.11\right)$. During follow-up, significant differences, with a moderate effect size, were only present in the DecreasedQoL group $\left(\eta^{2}=0.08\right)$ and the Increased-QoL group $\left(\eta^{2}=0.12\right)$. Complete data are shown in Table 3 .

Figures 1A and 1B show the change in QoL scores for the three groups in the QoL-Baseline and QoLChange models, respectively.

The proportion of patients classified as GDS 4, 5 and 6 did not change significantly in either the QoL-Baseline model (Baseline, $\chi^{2}=0.4, p=0.978$; 12 months, $\chi^{2}=1.4, p=0.835$; 24 months, $\chi^{2}=7.4$, $p=0.115$ ) or the QoL-Change model (Baseline, $\chi^{2}=6.6, p=0.153 ; 12$ months, $\chi^{2}=3.3, p=0.501 ; 24$ 

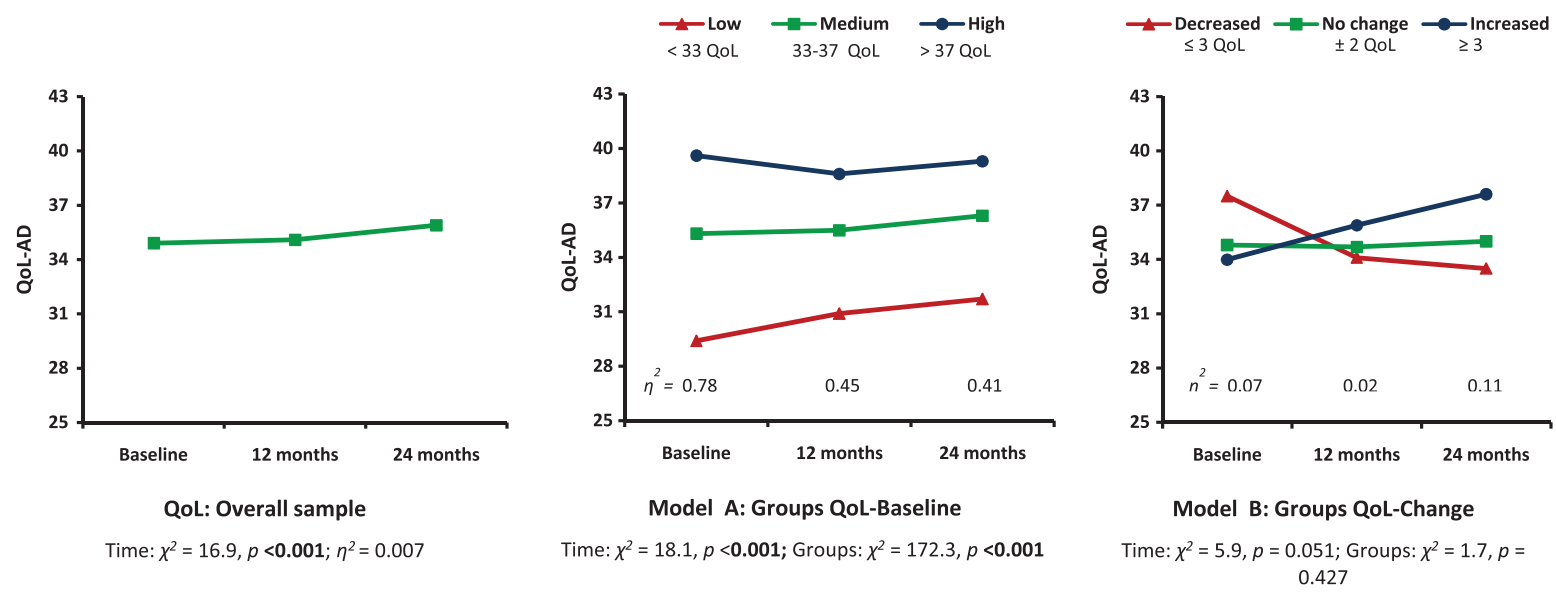

Fig. 1. Two models for analyzing QoL across follow up. Wald $\chi^{2}=$ Generalized estimating equations. $\eta^{2}=$ Eta-squared (effect size).

months, $\left.\chi^{2}=3.0, p=0.557\right)$. Similarly, there were no significant changes in either model (QoL-Baseline or QoL-Change) in the proportions of subjects according to age group $( \pm 77)$, gender, years of schooling ( \pm 5 years), type of family caregiver (spouse/adult child), or living with the caregiver.

\section{Clinical variables in the QoL-Baseline model during follow-up}

The data for the main variables in relation to the three subgroups formed using the QoL-Baseline model are shown in Table 4.

The effect of depression (GDS) was strongest in the Low-QoL group. There were important betweengroup differences at each assessment point $\left(\eta^{2}\right.$; $\mathrm{T} 1=0.26, \mathrm{~T} 2=0.24, \mathrm{~T} 3=0.26$ ), but in none of the groups was a significant change observed in this variable across follow-up. When depression was analyzed with the NPI (Depression subscale) the results obtained were consistent with those from the GDS: The Low-QoL group scored higher and the High-QoL group lower, although the effect sizes were smaller than those for the GDS $\left(\eta^{2} ; \mathrm{T} 1=0.13, \mathrm{~T} 2=0.09\right.$, $\mathrm{T} 3=0.10)$.

With respect to anosognosia the lowest scores corresponded to the Low-QoL group and the highest scores to the High-QoL group, the opposite of the effect observed for depression. Between-group differences increased across the three assessment points $\left(\eta^{2} ; \mathrm{T} 1=0.04, \mathrm{~T} 2=0.06, \mathrm{~T} 3=0.07\right)$. During followup, anosognosia increased in all three groups, most notably in the High-QoL group, although the effect sizes were weak $\left(\eta^{2}\right.$; Low $=0.01$, Medium $=0.02$, High = 0.04).
The greatest number of neuropsychiatric symptoms was presented by patients in the Low-QoL group. Between-group differences were only significant at 24 months, with a weak effect size $\left(\eta^{2}\right.$ $=0.049$ ). During follow-up, all the groups showed an increase in NPI scores, although the effect sizes were weak $\left(\eta^{2} ;\right.$ Low $=0.05$, Medium $=0.02$, High $\left.=0.04\right)$.

With respect to functional impairment the between-group differences were small, and only at baseline did the Low-QoL group score significantly lower $\left(\eta^{2}=0.04\right)$. By contrast, all three groups showed a notable deterioration across the follow-up $\operatorname{period}\left(\eta^{2} ;\right.$ Low $=0.12$, Medium $=0.16$, High $\left.=0.21\right)$, with the change being greatest in the High-QoL group.

The results for cognitive status were similar to those for functional ability: The between-group differences were not significant at any of the assessment points, but there was a moderate-to-large deterioration in cognitive function in all three groups across follow-up $\left(\eta^{2}\right.$; Low $=0.09$, Medium $=0.08$, High $=0.15$ ), with the greatest change corresponding to the High-QoL group.

\section{Clinical variables in the QoL-Change model during follow-up}

The data for the main variables in relation to the three subgroups formed using the QoL-Change model are shown in Supplementary Table 1.

Depression scores (GDS) were always lower in the Increased-QoL group, although the between-group differences were only significant, with a moderate effect size, at 24 months $\left(\eta^{2}=0.07\right)$. Only in the Increased-QoL group was a decrease in scores, with a 
Table 4

Groups according to the QoL-Baseline model. Scores for clinical variables

\begin{tabular}{|c|c|c|c|c|c|c|}
\hline & \multirow{2}{*}{$\begin{array}{l}\text { 1. Baseline } \\
\text { Mean (SE) }\end{array}$} & \multirow{2}{*}{$\begin{array}{c}\text { 2. } 12 \text { months } \\
\text { Mean (SE) }\end{array}$} & \multirow{2}{*}{$\begin{array}{c}3.24 \text { months } \\
\text { Mean (SE) }\end{array}$} & \multicolumn{3}{|c|}{ Simple effects } \\
\hline & & & & $\chi^{2}$ & $d f$ & $p$ \\
\hline \multicolumn{7}{|l|}{ Depression (GDS) } \\
\hline Low-QoL & $5.2(0.4)$ & $5.2(0.5)$ & $4.9(0.4)$ & 3.5 & 2 & 0.171 \\
\hline Medium & $3.3(0.3)^{\mathrm{d}}$ & $3.5(0.3)^{\mathrm{d}}$ & $3.3(0.3)^{\mathrm{d}}$ & 0.4 & 2 & 0.810 \\
\hline High-QoL & $1.6(0.2)^{\mathrm{e}, \mathrm{f}}$ & $1.7(0.2)^{\mathrm{e}, \mathrm{f}}$ & $1.3(0.2)^{\mathrm{e}, \mathrm{f}}$ & 5.3 & 2 & 0.069 \\
\hline Simple effects $\chi^{2}(d f) p$ & $49.2(2)<\mathbf{0 . 0 0 1}$ & $46.6(2)<0.001$ & $47.0(2)<\mathbf{0 . 0 0 1}$ & & & \\
\hline - Model $\chi^{2}(d f) p$ & \multicolumn{6}{|c|}{ Time: 4.0 (2) 0.132 ; Groups: 58.6 (2)<0.001; Time*groups: 0.9 (4) 0.913} \\
\hline \multicolumn{7}{|l|}{ Depression (NPI) } \\
\hline Low-QoL & $3.2(0.5)$ & $3.7(0.5)$ & $3.9(0.6)$ & 1.1 & 2 & 0.569 \\
\hline Medium & $3.1(0.4)$ & $2.6(0.5)$ & $1.6(0.3)^{\mathrm{c}, \mathrm{d}}$ & 11.3 & 2 & 0.003 \\
\hline High-QoL & $1.0(0.2)^{\mathrm{e}, \mathrm{f}}$ & $1.2(0.3)^{\mathrm{e}}$ & $1.4(0.5)^{\mathrm{e}}$ & 1.0 & 2 & 0.587 \\
\hline Simple effects $\chi^{2}(d f) p$ & $26.0(2)<\mathbf{0 . 0 0 1}$ & 13.9 (2) 0.001 & $12.0(2) \mathbf{0 . 0 0 2}$ & & & \\
\hline - Model $\chi^{2}(d f) p$ & \multicolumn{6}{|c|}{ Time: 0.2 (2) 0.863; Groups: 22.6 (2)<0.001; Time* groups: 12.0 (4) $\mathbf{0 . 0 1 7}$} \\
\hline \multicolumn{7}{|l|}{ Anosognosia (AQ-D) } \\
\hline Low-QoL & $26.5(2.8)$ & $30.5(3.1)^{\mathrm{a}}$ & $32.6(2.9)^{\mathrm{b}}$ & 14.4 & 2 & 0.001 \\
\hline Medium & $28.4(2.7)$ & $31.5(3.0)$ & $36.2(2.7)^{\mathrm{b}, \mathrm{c}}$ & 13.4 & 2 & 0.001 \\
\hline High-QoL & $35.5(2.5)$ & $40.9(2.3)^{\mathrm{a}, \mathrm{e}, \mathrm{f}}$ & $44.4(2.3)^{\mathrm{b}, \mathrm{c}, \mathrm{e}}$ & 19.2 & 2 & $<0.001$ \\
\hline Simple effects $\chi^{2}(d f) p$ & $6.3(2) \mathbf{0 . 0 4 2}$ & 9.4 (2) 0.009 & 10.9 (2) 0.004 & & & \\
\hline - Model $\chi^{2}(d f) p$ & \multicolumn{6}{|c|}{ Time: 45.1 (2)<0.001; Groups: 10.1 (2) $\mathbf{0 . 0 0 6}$; Time* groups: 3.0 (4) 0.555} \\
\hline \multicolumn{7}{|l|}{ Behavior (NPI) } \\
\hline Low-QoL & $24.3(2.7)$ & $28.0(2.7)$ & $34.4(2.8)^{\mathrm{b}}$ & 8.8 & 2 & 0.012 \\
\hline Medium & $21.3(2.3)$ & $20.4(2.2)$ & $26.1(2.9)^{\mathrm{c}}$ & 7.3 & 2 & 0.025 \\
\hline High-QoL & $17.5(2.3)$ & $22.2(2.3)$ & $25.8(2.2)^{\mathrm{b}}$ & 15.8 & 2 & $<0.001$ \\
\hline Simple effects $\chi^{2}(d f) p$ & $3.6(2) 0.158$ & 4.7 (2) 0.092 & $6.4(2) 0.039$ & & & \\
\hline - Model $\chi^{2}(d f) p$ & \multicolumn{6}{|c|}{ Time: 25.2 (2) < $\mathbf{0 . 0 0 1}$; Groups: 6.7 (2) $\mathbf{0 . 0 3 5}$; Time* groups: 3.9 (4) 0.415} \\
\hline \multicolumn{7}{|l|}{ Function (DAD) } \\
\hline Low-QoL & $57.5(1.4)$ & $52.6(1.5)^{\mathrm{a}}$ & $48.8(1.4)^{\mathrm{b}, \mathrm{c}}$ & 66.6 & 2 & $<0.001$ \\
\hline Medium & $62.5(1.5)$ & $56.9(1.6)^{\mathrm{a}}$ & $51.2(1.4)^{\mathrm{b}, \mathrm{c}}$ & 67.2 & 2 & $<\mathbf{0 . 0 0 1}$ \\
\hline High-QoL & $61.5(1.4)$ & $56.7(1.2)^{\mathrm{a}}$ & $49.9(1.3)^{\mathrm{b}, \mathrm{c}}$ & 82.4 & 2 & $<0.001$ \\
\hline Simple effects $\chi^{2}(d f) p$ & $6.3(2) \mathbf{0 . 0 4 2}$ & 4.9 (2) 0.082 & $1.3(2) 0.506$ & & & \\
\hline - Model $\chi^{2}(d f) p$ & \multicolumn{6}{|c|}{ Time: 209.6 (2)<0.001; Groups: 4.3 (2) 0.112 ; Time* groups: 8.9 (4) 0.063} \\
\hline \multicolumn{7}{|l|}{ Cognition (MMSE) } \\
\hline Low-QoL & $17.7(0.8)$ & $15.4(1.0)^{\mathrm{a}}$ & $12.5(1.1)^{\mathrm{b}, \mathrm{c}}$ & 39.9 & 2 & $<0.001$ \\
\hline Medium & $19.4(0.7)$ & $16.9(0.8)^{\mathrm{a}}$ & $15.0(0.9)^{\mathrm{b}, \mathrm{c}}$ & 38.3 & 2 & $<0.001$ \\
\hline High-QoL & $20.1(0.7)$ & $18.0(0.7)^{\mathrm{a}}$ & $14.3(1.0)^{\mathrm{b}, \mathrm{c}}$ & 34.0 & 2 & $<0.001$ \\
\hline Simple effects $\chi^{2}(d f) p$ & $4.5(2) 0.101$ & $4.1(2) 0.123$ & $2.7(2) 0.255$ & & & \\
\hline - Model $\chi^{2}(d f) p$ & \multicolumn{6}{|c|}{ Time: 108.5 (2)<0.001; Groups: 3.8 (2) 0.148 ; Time* groups: 6.1 (4) 0.189} \\
\hline
\end{tabular}

Generalized estimating equations, Wald $\chi^{2}$; Means, estimated marginal; SE, standard error; Significant with Bonferroni post hoc contrasts: ${ }^{\mathrm{a}} 1-2,{ }^{\mathrm{b}} 1-3,{ }^{\mathrm{c}} 2-3$, ${ }^{\mathrm{d}}$ Low-Medium, ${ }^{\mathrm{e}}$ Low-High, ${ }^{\mathrm{f}}$ Medium-High. $p$-values $<0.05$ are shown in bold. QoL-AD, Quality of Life in Alzheimer's Disease scale; AQ-D, Anosognosia Questionnaire-Dementia; GDS, Geriatric Depression Scale; NPI, Neuropsychiatric Inventory; DAD, Disability Assessment in Dementia; MMSE, Mini-Mental State Examination.

weak effect size $\left(\eta^{2}=0.01\right)$, observed during followup. When depression was analyzed with the NPI (Depression subscale) the results were consistent with those obtained from the GDS: The IncreasedQoL group had the lowest scores. However, the differences between groups were not significant at any of the assessment points, and the effect sizes were small $\left(\eta^{2} ; \mathrm{T} 1=0.01, \mathrm{~T} 2=0.03, \mathrm{~T} 3=0.01\right)$.

With respect to anosognosia the highest scores corresponded to the Increased-QoL group and the lowest scores to the Decreased-QoL group, although the between-group differences only showed a moderate effect at 24 months $\left(\eta^{2}=0.06\right)$. During follow-up, anosognosia increased in all the groups, although the effect sizes were weak $\left(\eta^{2}\right.$; Decreased $=0.00$, No change $=0.02$, Increased $=0.04$ ).

The greatest number of neuropsychiatric symptoms was presented by patients in the Increased-QoL group, although between-group differences were not significant. Across follow-up a significant change, with a weak effect size, was only observed in the No change $\left(\eta^{2}=0.04\right)$ and the Increased-QoL $\left(\eta^{2}=0.03\right)$ groups.

With respect to functional impairment the between-group differences were not significant at any of the assessment points, but each of the three groups showed a notable deterioration across follow-up $\left(\eta^{2} ;\right.$ Decreased $=0.19$, No Change $=0.15$, 
Table 5

Clinical variables. Multinomial logistic regression analysis at baseline assessment

\begin{tabular}{|c|c|c|c|c|c|c|}
\hline Method: Enter together & $\mathrm{B}$ & (SE) & Wald & $p$ & OR & $95 \% \mathrm{CI}$ \\
\hline \multicolumn{7}{|l|}{ A. Groups QoL-Baseline } \\
\hline \multicolumn{7}{|l|}{ Low QoL $(<33$ QoL $)$} \\
\hline Depression (GDS) & 0.18 & $(0.09)$ & 3.89 & 0.048 & 1.20 & $1.00-1.45$ \\
\hline Anosognosia (AQ-D) & -0.04 & $(0.02)$ & 3.71 & 0.054 & 0.95 & $0.91-1.00$ \\
\hline Function (DAD) & -0.08 & $(0.03)$ & 4.57 & $\mathbf{0 . 0 3 2}$ & 0.92 & $0.85-0.99$ \\
\hline Behavior (NPI) & 0.01 & $(0.01)$ & 0.92 & 0.335 & 1.01 & $0.98-1.05$ \\
\hline Cognition (MMSE) & -0.04 & $(0.05)$ & 0.63 & 0.426 & 0.95 & $0.86-1.06$ \\
\hline \multicolumn{7}{|l|}{ High QoL (>37 QoL) } \\
\hline Depression (GDS) & -0.38 & $(0.13)$ & 8.29 & 0.004 & 0.68 & $0.52-0.88$ \\
\hline Anosognosia (AQ-D) & 0.06 & $(0.02)$ & 6.28 & 0.012 & 1.07 & $1.01-1.13$ \\
\hline Function (DAD) & 0.02 & $(0.04)$ & 0.40 & 0.524 & 1.02 & $0.94-1.11$ \\
\hline Behavior (NPI) & -0.04 & $(0.02)$ & 5.74 & 0.017 & 0.95 & $0.91-0.99$ \\
\hline Cognition (MMSE) & 0.08 & $(0.05)$ & 2.65 & 0.103 & 1.08 & $0.98-1.20$ \\
\hline \multicolumn{7}{|c|}{ Reference group: Medium QoL (33-37 QoL) } \\
\hline \multicolumn{7}{|c|}{ B. Groups QoL-Change } \\
\hline \multicolumn{7}{|l|}{ Decreased ( $\leq 3$ QoL) } \\
\hline Depression (GDS) & -0.09 & $(0.10)$ & 0.89 & 0.344 & 0.90 & $0.74-1.10$ \\
\hline Anosognosia (AQ-D) & -0.01 & $(0.02)$ & 0.27 & 0.597 & 0.98 & $0.94-1.03$ \\
\hline Function (DAD) & 0.04 & $(0.04)$ & 0.96 & 0.327 & 1.04 & $0.96-1.13$ \\
\hline Behavior (NPI) & 0.03 & $(0.02)$ & 1.98 & 0.159 & 1.03 & $0.98-1.07$ \\
\hline Cognition (MMSE) & 0.03 & $(0.05)$ & 0.37 & 0.540 & 1.03 & $0.92-1.15$ \\
\hline \multicolumn{7}{|l|}{ Increased ( $\geq 3$ QoL) } \\
\hline Depression (GDS) & -0.18 & $(0.08)$ & 4.69 & $\mathbf{0 . 0 3 0}$ & 0.83 & $0.70-0.98$ \\
\hline Anosognosia (AQ-D) & -0.00 & $(0.01)$ & 0.24 & 0.624 & 0.99 & $0.95-1.02$ \\
\hline Function (DAD) & 0.00 & $(0.03)$ & 0.02 & 0.866 & 1.00 & $0.94-1.06$ \\
\hline Behavior (NPI) & 0.02 & $(0.01)$ & 1.40 & 0.236 & 1.02 & $0.98-1.05$ \\
\hline Cognition (MMSE) & -0.02 & $(0.04)$ & 0.28 & 0.595 & 0.97 & $0.89-1.06$ \\
\hline \multicolumn{7}{|c|}{ Reference group: No change QoL ( \pm 2 QoL) } \\
\hline
\end{tabular}

B, unstandardized coefficient; SE, standard error; OR, odds ratio; CI, confidence interval QoL-AD, Quality of Life in Alzheimer's Disease scale; AQ-D, Anosognosia Questionnaire-Dementia; GDS, Geriatric Depression Scale; NPI, Neuropsychiatric Inventory; DAD, Disability Assessment in Dementia; MMSE, Mini-Mental State Examination.

Increased $=0.17$ ). The results for cognitive status were similar to those for functional ability: No significant between-group differences were observed, but there was a moderate-to-large deterioration in cognitive function in all three groups across follow-up $\left(\eta^{2}\right.$; Decreased $=0.22$, No change $=0.06$, Increased $=0.12$ ).

\section{Multinomial regression analysis of the groups of patients at baseline assessment}

In the multinomial regression we took the intermediate group in each model (i.e. Medium in the QoL-Baseline model and No change in the QoLChange model) as the reference group (Table 5).

In the QoL-Baseline model the predictors for the Low-QoL group were greater depression and poorer functional ability. In the High-QoL group the predictors were less depression, more anosognosia, and fewer neuropsychiatric symptoms.

In the QoL-Change model there were no significant predictors for the Decreased-QoL group, and only less depression was a predictor in the Increased-QoL group.

\section{DISCUSSION}

Factors associated with QoL in the sample as a whole

Depression, neuropsychiatric symptoms, anosognosia, and functional ability were all shown to be relevant when analyzing the overall sample, although the most important factors were anosognosia and depression.

The association between greater depression and lower scores on self-perceived QoL has been widely reported in longitudinal and cross-sectional studies of community samples [5, 6, 8, 11, 18, 34-38] and of patients attending day centers [39] or living in residential care $[2,4,40]$. Furthermore, the results obtained have been similar despite the variety of instruments used to assess depression: Cornell Scale for Depression in Dementia (CSDD) [2, 4], Center for 
Epidemiologic Studies-Depression Scale (CES-D) [6], Hospital Anxiety and Depression Scale (HADS) [8], NPI-Depression [5, 11,37], Geriatric Depression Scale (GDS) [18, 36, 38, 39], Hamilton Depression Scale (HRSD) [35], and the Beck Depression Inventory (BDI) [34].

Although depression is the neuropsychiatric symptom most widely reported to be associated with poorer QoL, this association with impaired QoL has also been found for the total NPI score [5, 11, 17], as well as for other specific neuropsychiatric symptoms, including apathy [39], anxiety [2, 4], agitation [40], and psychotic symptoms assessed by the NPI (Delusions, Hallucinations) [5].

The association between greater anosognosia and a more positive view of QoL among patients with dementia has been well documented in both crosssectional [41-45] and longitudinal studies [10, 11, $18]$.

Finally, some studies have also reported an association between better functional ability and higher self-ratings of QoL [3, 6].

\section{Anosognosia, depression, and quality of life}

The relationship between anosognosia and depression, however, requires a more detailed analysis. The first authors to report an inverse relationship between anosognosia and depression were Sevush and Leve [46]. Subsequent research confirmed this inverse association, suggesting that depression is more likely to be present when patients have a greater awareness of their deficits (less anosognosia), and therefore that it is a reaction to the process of deterioration [25, 47-49].

The combination of these two factors and their relationship to perceived QoL has also been investigated, with similar findings being obtained: Greater anosognosia was associated with less depression and a more positive view of QoL, and vice-versa, that is, poorer self-perceived QoL was linked to more depression and less anosognosia $[11,18,50,51]$.

\section{Comparison of the QoL-Baseline and QoL-Change models}

Focusing on the baseline scores in the two models the QoL-Baseline model showed greater betweengroup variability (better differentiation between groups) and less within-group variability (greater homogeneity of subjects in each group) than did the QoL-Change model. It should also be noted that cer- tain clinical factors (depression, functional ability, and neuropsychiatric symptoms) were shown to be significantly associated with this between-group variability in the QoL-Baseline model. These results support our initial hypothesis and the findings of previous studies in community samples $[6,7,9]$ regarding the superiority of the QoL-Baseline model for the analysis of data, as well as the good predictive ability of the baseline QoL score.

In addition, the analysis of the QoL-Baseline model showed that the influence of the clinical variables differed according to the groups. Depression was greater in the Low-QoL group and lower in the High-QoL group. The opposite pattern was observed for anosognosia, that is, scores were lower in the LowQoL group and higher in the High-QoL group. These distributions were the same at all three assessment points. The Low-QoL group also presented more neuropsychiatric symptoms and poorer functional ability. The analysis of the QoL-Change model showed less variability between the groups formed according to this model. The only notable finding was that the Increased-QoL group presented less depression and greater anosognosia, although this was only the case at 24 months.

It should be noted that when scores on the NPI Depression subscale were used as a measure of depression in the groups of both models, the results were consistent with those obtained with the GDS, although their statistical relevance was reduced. This finding is in line with previous research [39]. Depression is clearly the most important clinical factor associated with poorer self-perceived QoL in both models (Low-QoL group and Decreased-QoL group), although the statistical effect is more notable in the QoL-Baseline model.

The results of this study confirm that the selfrating of $\mathrm{QoL}$ at baseline (Low, High) is more relevant than the change in QoL ratings over time (Decreased, Increased). Although a number of previous studies $[2,4,9,14]$ have used a model based on deterioration-stability-improvement in QoL scores across follow-up, the model based on the baseline QoL score seems to offer certain advantages. From a psychometric point of view the QoL-Baseline model was a better predictor of perceived QoL at 24 months, and it also enabled us to observe the effect of the different variables in each group. This model would likewise be useful from a clinical point of view. Given that QoL scores have been shown to remain relatively stable over time in both community samples [3, 5-11] and residential populations $[2,4,14]$, the baseline 
score could be used to predict how a patient is likely to evolve, thus enabling suitable interventions to be targeted at the factors known to influence QoL, especially depression and anosognosia/cognitive status.

\section{Clinical implications}

The results of this study suggest that a longitudinal analysis of self-perceived QoL among patients with AD should use a model in which groups are created according to baseline scores, since this model is better than one based on a change in scores when it comes to identifying trajectories and predictors of QoL. This recommendation follows from the fact that the key feature of QoL scores is not change over time but, rather, the stability of the different score ranges.

In terms of intervention, the assessment of a patient's baseline status, covering depression, cognitive and functional ability, and neuropsychiatric symptoms, should serve as a platform on which to implement strategies that can help ameliorate the negative effects of the condition, especially among patients with poor perceived QoL. There is an extensive literature on non-pharmacological interventions in patients with dementia, although they appear to have only a moderate effect on the different variables involved. A recent review of six randomized controlled studies found beneficial effects with respect to depression and anxiety symptoms [52]. Music therapy has also been shown to have some benefits with regard to reducing neuropsychiatric symptoms $[53,54]$, improving symptoms of anxiety and depression $[55,56]$, enhancing cognitive performance and quality of life [55, 57], and even for improving or stabilizing certain aspects of self-awareness [58]. Other studies have found that cognitive stimulation therapy can improve cognition [59, 60] and quality of life [59, 61], reducing apathy and symptoms of depression. Reminiscence programs appear to improve symptoms of depression and interpersonal relationships [62, 63]. Physical exercise reduced neuropsychiatric symptoms in patients with mild dementia [64]. A program of therapeutic education for patients and caregivers improve the quality of life [65]. Finally, multicomponent programs have been shown to improve apathy, anxiety, depression, and quality of life [66].

\section{Limitations and future research}

The main limitation of this study is that depression and anosognosia were not assessed clinically, the data being based solely on self- and informant report measures.

Our results are unlikely to have been seriously affected by the loss of some cases to follow-up, since QoL scores do not appear to be influenced by the severity of dementia.

Future research should nonetheless aim to replicate the present analysis with patients in residential care facilities, since, although the severity of dementia was not shown here to have an effect on perceived QoL, it is possible that community and institutionalized samples would yield different results. Indeed, whereas all research in community samples reports stability of QoL scores over follow-up [3, 5-13, 18], these scores have been reported to decrease over time in some residential samples [15-17], probably due to increased severity of dementia in these patients. It would also be interesting to examine possible changes in QoL related to pharmacological and non-pharmacological interventions, and also to analyze in greater depth the variability associated with family and contextual factors.

\section{ACKNOWLEDGMENTS}

This study was conducted within the framework of the project: Assessing perceptions of patient quality of life in patients with Alzheimer's disease and their family caregivers over a two-year period, funded by Spain's Ministry of Economy and Competitiveness (ref: PSI2010-19014). PI: Conde-Sala JL.

Authors' disclosures available online (http://jalz.com/manuscript-disclosures/16-0040r1).

\section{SUPPLEMENTARY MATERIAL}

The supplementary material is available in the electronic version of this article: http://dx.doi.org/ 10.3233/JAD-160040.

\section{REFERENCES}

[1] World Health, Organization (2012) Dementia: A public health priority. WHO Press, Geneva. http://www.who.int/ mental_health/publications/dementia_report_2012/en/index. html. Accessed on October 29, 2015.

[2] Selwood A, Thorgrimsen L, Orrell M (2005) Quality of life in dementia-a one-year follow-up study. Int J Geriatr Psychiatry 20, 232-237.

[3] Missotten P, Ylieff M, Di Notte D, Paquay L, De Lepeleire J, Buntinx F, Fontaine O (2007) Quality of life in dementia: A 2-year follow-up study. Int J Geriatr Psychiatry 22, 12011207. 
[4] Hoe J, Hancock G, Livingston G, Woods B, Challis D, Orrell M (2009) Changes in the quality of life of people with dementia living in care homes. Alzheimer Dis Assoc Disord 23, 285-290.

[5] Tatsumi H, Nakaaki S, Torii K, Shinagawa Y, Watanabe N, Murata Y, Sato J, Mimura M, Furukawa TA (2009) Neuropsychiatric symptoms predict change in quality of life of Alzheimer disease patients: A two-year follow-up study. Psychiatry Clin Neurosci 63, 374-384.

[6] Heggie M, Morgan D, Crossley M, Kirk A, Wong P, Karunanayake C, Beever R (2012) Quality of life in early dementia: Comparison of rural patient and caregiver ratings at baseline and one year. Dementia (London) 11, 521-541.

[7] Livingston G, Cooper C, Woods J, Milne A, Katona C (2012) Successful ageing in adversity: The LASER-AD longitudinal study. J Neurol Neurosurg Psychiatry 79, 641-645.

[8] Bosboom PR, Alfonso H, Almeida OP (2013) Determining the predictors of change in quality of life self-ratings and carer-ratings for community-dwelling people with Alzheimer disease. Alzheimer Dis Assoc Disord 27, 363371.

[9] Clare L, Woods RT, Nelis SM, Martyr A, Marková IS, Roth I, Whitaker CJ, Morris RG (2014) Trajectories of quality of life in early-stage dementia: Individual variations and predictors of change. Int J Geriatr Psychiatry 29, 616-623.

[10] Conde-Sala JL, Turró-Garriga O, Garre-Olmo J, VilaltaFranch J, López-Pousa S (2014) Discrepancies regarding the quality of life of patients with Alzheimer's disease: A three-year longitudinal study. J Alzheimers Dis 39, 511-525.

[11] Conde-Sala JL, Turró-Garriga O, Piñan-Hernández S, Portellano-Ortiz C, Viñas-Diez V, Gascón-Bayarri J, Reñé-Ramírez R (2016) Effects of anosognosia and neuropsychiatric symptoms on the quality of life of patients with Alzheimer's disease: A 24-month follow-up study. Int J Geriatr Psychiatr 31, 109-119.

[12] Lacey L, Bobula J, Rüdell K, Alvir J, Leibman C (2015) Quality of life and utility measurement in a large clinical trial sample of patients with mild to moderate Alzheimer's disease: Determinants and level of changes observed. Value Health 18, 638-645.

[13] Trigg R, Jones RW, Knapp M, King D, Lacey LA (2015) The relationship between changes in quality of life outcomes and progression of Alzheimer's disease: Results from the Dependence in $\mathrm{AD}$ in England 2 longitudinal study. Int $J$ Geriatr Psychiatr 30, 400-408.

[14] Beerens HC, Zwakhalen SM, Verbeek H, Ruwaard D, Ambergen AW, Leino-Kilpi H, Stephan A, Zabalegui A, Soto M, Saks K, Bökberg C, Sutcliffe CL, Hamers JP (2015) Change in quality of life of people with dementia recently admitted to long-term care facilities. $J$ Adv Nurs 71, 14351447.

[15] Lyketsos CG, Gonzales-Salvador T, Chin JJ, Baker A, Black B, Rabins P (2003) A follow-up study of change in quality of life among persons with dementia residing in a long-term care facility. Int J Geriatr Psychiatr 18, 275-281.

[16] Castro-Monteiro E, Forjaz MJ, Ayala A, RodriguezBlazquez C, Fernandez-Mayoralas G, Diaz-Redondo A, Martínez-Martín P (2014) Change and predictors of quality of life in institutionalized older adults with dementia. Qual Life Res 23, 2595-2601.

[17] Mjørud M, Røsvik J, Rokstad AM, Kirkevold M, Engedal K (2014) Variables associated with change in quality of life among persons with dementia in nursing homes: A 10 months follow-up study. PLoS One 9, e115248.
[18] Portellano-Ortiz C, Turró-Garriga O, Gascón-Bayarri J, Piñán-Hernández S, Moreno-Cordón L, Viñas-Diez V, Reñe-Ramírez R, Conde-Sala JL (2014) The influence of anosognosia and depression on the perceived quality of life of patients with Alzheimer's disease: A 12 months followup. Rev Neurol 59, 193-204.

[19] Bosboom PR, Almeida OP (2014) Do changes in specific cognitive functions predict changes in health-related quality of life in people with Alzheimer's disease? Int J Geriatr Psychiatr 29, 694-703.

[20] American Psychiatric Association (2013) Diagnostic and Statistical Manual of Mental Disorders, DSM-5. APA Press, Washington DC.

[21] McKhann G, Drachman D, Folstein M, Katzman R, Price D, Stadlan EM (1984) Clinical diagnosis of Alzheimer's disease: Report of the NINCDS-ADRDA Work Group under the auspices of Department of Health and Human Services task force on Alzheimer's disease. Neurology 34, 939-944.

[22] Folstein MF, Folstein SE, McHugh PR (1975) “Mini Mental State". A practical method for grading the cognitive state of patients for the clinician. J Psychiatr Res 12, 189-198.

[23] Logsdon RG, Gibbons LE, McCurry SM, Teri L (2002) Assessing quality of life in older adults with cognitive impairment. Psychosom Med 64, 510-519.

[24] Cummings JL, Mega M, Gray K, Rosemberg-Thompson S, Carusi DA, Gornbein J (1994) The neuropsychiatric inventory. Comprehensive assessment of psychopathology in dementia. Neurology 44, 2308-2314.

[25] Migliorelli R, Tesón A, Sabe L, Petracca G, Petracchi M, Leiguarda R, Starkstein SE (1995) Anosognosia in Alzheimer's disease: A study of associated factors. J Neuropsychiatry Clin Neurosci 7, 338-344.

[26] Blesa R, Pujol M, Aguilar M, Santacruz P, Bertran-Serra I, Hernández G, Sol JM, Peña-Casanova J (2001) Clinical validity of the 'mini-mental state' for Spanish speaking communities. Neuropsychologia 39, 1150-1157.

[27] Sheikh JI, Yesavage JA (1986) Geriatric Depression Scale (GDS): Recent evidence and development of a shorter version. Clin Gerontol 5, 165-173.

[28] Votruba KL, Persad C, Giordani B (2015) Patient mood and instrumental activities of daily living in Alzheimer disease: Relationship between patient and caregiver reports. J Geriatr Psychiatry Neurol 28, 203-209.

[29] D'Onofrio G, Sancarlo D, Addante F, Ciccone F, Cascavilla L, Paris F, Elia AC, Nuzzaci C, Picoco M, Greco A, Panza F, Pilotto A (2015) A pilot randomized controlled trial evaluating an integrated treatment of rivastigmine transdermal patch and cognitive stimulation in patients with Alzheimer's disease. Int J Geriatr Psychiatr 30, 965-975.

[30] Gélinas I, Gauthier L, McIntyre M, Gauthier S (1999) Development of a functional measure for persons with Alzheimer's disease: The Disability Assessment for Dementia. Am J Occup Ther 53, 471-481.

[31] Reisberg B, Ferris SH, De Leon MJ, Crook T (1982) The Global Deterioration Scale for assessment of primary degenerative dementia. Am J Psychiatry 139, 1136-1139.

[32] Liang K, Zeger SL (1986) Longitudinal data analysis using generalized linear models. Biometrika 73, 13-22.

[33] Cohen J (1973) Eta-squared and partial eta-squared in fixed factor ANOVA designs. Educ Psychol Meas 33, 107-112.

[34] Karttunen K, Karppi P, Hiltunen A, Vanhanen M, Välimäki T, Martikainen J, Valtonen H, Sivenius J, Soininen H, Hartikainen S, Suhonen J, Pirttilä T (2011) Neuropsychiatric symptoms and quality of life in patients with very mild 
and mild Alzheimer's disease. Int J Geriatr Psychiatry 26, 473-482.

[35] Li M, Huang H, Jiang G, Mou X, Chen Q (2015) Change patterns, influencing factors and predictors of quality of life in patients with Alzheimer's disease. Zhonghua Yi Xиe Za Zhi 95, 1131-1134.

[36] Naglie G, Hogan DB, Krahn M, Beattie BL, Black SE, Macknight C, Freedman M, Patterson C, Borrie M, Bergman H, Byszewski A, Streiner D, Irvine J, Ritvo P, Comrie J, Kowgier M, Tomlinson G (2011) Predictors of patient self-ratings of quality of life in Alzheimer disease: Cross-sectional results from the Canadian Alzheimer's Disease Quality of Life Study. Am J Geriatr Psychiatry 19, 881-890.

[37] Shin IS, Carter M, Masterman D, Fairbanks L, Cummings JL (2005) Neuropsychiatric symptoms and quality of life in Alzheimer disease. Am J Geriatr Psychiatry 13, 469-474.

[38] Vogel A, Mortensen EL, Hasselbalch SG, Andersen BB, Waldemar G (2006) Patient versus informant reported quality of life in the earliest phases of Alzheimer's disease. Int J Geriatr Psychiatry 21, 1132-1138.

[39] Gómez-Gallego M, Gómez-Amor J, Gómez-García J (2012) Determinants of quality of life in Alzheimer's disease: Perspective of patients, informal caregivers, and professional caregivers. Int Psychogeriatr 24, 1805-1815.

[40] Beerens HC, Zwakhalen SM, Verbeek H, Ruwaard D, Hamers JP (2013) Factors associated with quality of life of people with dementia in long-term care facilities: A systematic review. Int J Nurs Stud 50, 1259-1270.

[41] Trigg R, Watts S, Jones R, Tod A (2011) Predictors of quality of life ratings from persons with dementia: The role of insight. Int J Geriatr Psychiatry 26, 83-91.

[42] Ready RE, Ott BR, Grace J (2006) Insight and cognitive impairment: Effects on quality-of-life reports from mild cognitive impairment and Alzheimer's disease patients. Am $J$ Alzheimers Dis Other Demen 21, 242-248.

[43] Berwig M, Leicht H, Gertz HJ (2009) Critical evaluation of self-rated quality of life in mild cognitive impairment and Alzheimer's disease-further evidence for the impact of anosognosia and global cognitive impairment. J Nutr Health Aging 13, 226-230.

[44] Hurt CS, Banerjee S, Tunnard C, Whitehead DL, Tsolaki M, Mecocci P, Kloszewska I, Soininen H, Vellas B, Lovestone $S$ (2010) Insight, cognition and quality of life in Alzheimer's disease. J Neurol Neurosurg Psychiatry 81, 331-336.

[45] Conde-Sala JL, Reñé-Ramírez R, Turró-Garriga O, GascónBayarri J, Campdelacreu-Fumadó J, Juncadella-Puig M, Rico-Pons I, Garre-Olmo J (2014) Severity of dementia, anosognosia and depression in relation to the quality of life of patients with Alzheimer's disease: Discrepancies between patients and caregivers. Am J Geriatr Psychiatry 22, 138-147.

[46] Sevush S, Leve N (1993) Denial of memory deficit in Alzheimer's disease. Am J Psychiatry 150, 748-751.

[47] Starkstein SE, Chemerinski E, Sabe L, Kuzis G, Petracca G, Tesón A, Leiguarda R (1997) Prospective longitudinal study of depression and anosognosia in Alzheimer's disease. $\mathrm{Br} \mathrm{J}$ Psychiatry 171, 47-52.

[48] Harwood DG, Sultzer DL, Wheatley MV (2000) Impaired insight in Alzheimer disease: Association with cognitive deficits, psychiatric symptoms, and behavioral disturbances. Neuropsychiatry Neuropsychol Behav Neurol 13, 83-88.

[49] Kashiwa Y, Kitabayashi Y, Narumoto J, Nakamura K, Ueda H, Fukui K (2005) Anosognosia in Alzheimer's disease:
Association with patient characteristics, psychiatric symptoms and cognitive deficits. Psychiatry Clin Neurosci 59, 697-704.

[50] Conde-Sala JL, Reñé-Ramírez R, Turró-Garriga O, Gascón-Bayarri J, Juncadella-Puig M, Moreno-Cordón L, Viñas-Díez L, Garre-Olmo J (2013) Clinical differences in patients with Alzheimer's disease according to the presence or absence of anosognosia: Implications for perceived quality of life. J Alzheimers Dis 33, 1105-1116.

[51] Sousa MF, Santos RL, Arcoverde C, Simões P, Belfort T, Adler I, Leal C, Dourado MC (2013) Quality of life in dementia: The role of non-cognitive factors in the ratings of people with dementia and family caregivers. Int Psychogeriatr 25, 1097-1105.

[52] Orgeta V, Qazi A, Spector AE, Orrell M (2014) Psychological treatments for depression and anxiety in dementia and mild cognitive impairment. Cochrane Database Syst Rev 1, CD009125.

[53] Sakamoto M, Ando H, Tsutou A (2013) Comparing the effects of different individualized music interventions for elderly individuals with severe dementia. Int Psychogeriatr 25, 775-784.

[54] Raglio A, Bellandi D, Baiardi P, Gianotti M, Ubezio MC, Zanacchi E, Granieri E, Imbriani M, Stramba-Badiale M (2015) Effect of active music therapy and individualized listening to music on dementia: A multicenter randomized controlled trial. J Am Geriatr Soc 63, 1534-1539.

[55] Blackburn R, Bradshaw T (2014) Music therapy for service users with dementia: A critical review of the literature. J Psychiatric Ment Health Nurs 21, 879-888.

[56] Guetin S, Portet F, Picot MC, Defez C, Pose C, Blayac JP, Touchon J (2009) Impact of music therapy on anxiety and depression for patients with Alzheimer's disease and on the burden felt by the main caregiver (feasibility study). Encephale 35, 57-65.

[57] Särkämö T, Laitinen S, Numminen A, Kurki M, Johnson JK, Rantanen P (2015) Clinical and demographic factors associated with the cognitive and emotional efficacy of regular musical activities in dementia. J Alzheimers Dis $\mathbf{4 9}$, 767-781.

[58] Arroyo-Anlló EM, Díaz JP, Gil R (2013) Familiar music as an enhancer of self-consciousness in patients with Alzheimer's disease. Biomed Res Int 2013, 752965.

[59] Knapp M, Thorgrimsen L, Patel A, Spector A, Hallam A, Woods B, Orrell M (2006) Cognitive stimulation therapy for people with dementia: Cost-effectiveness analysis. $\mathrm{Br} \mathrm{J}$ Psychiatry 188, 574-580.

[60] Spector A, Thorgrimsen L, Woods B, Royan L, Davies S, Butterworth M, Orrell M (2003) Efficacy of an evidencebased cognitive stimulation therapy programme for people with dementia: Randomised controlled trial. Br J Psychiatry 183, 248-254.

[61] Cooper C, Mukadam N, Katona C, Lyketsos CG, Ames D, Rabins P, Engedal K, de Mendonça Lima C, Blazer D, Teri L, Brodaty H, Livingston G (2012) Systematic review of the effectiveness of non-pharmacological interventions to improve quality of life of people with dementia. Int Psychogeriatr 24, 856-870.

[62] Gonzalez J, Mayordomo T, Torres M, Sales A, Meléndez JC (2015) Reminiscence and dementia: A therapeutic intervention. Int Psychogeriatr 27, 1731-1737.

[63] Serrani-Azcurra DJ (2012) A reminiscence program intervention to improve the quality of life of long-term care residents with Alzheimer's disease: A randomized controlled trial. Rev Bras Psiquiatr 34, 422-433. 
[64] Hoffmann K, Sobol NA, Frederiksen KS, Beyer N, Vogel A, Vestergaard K, Brændgaard H, Gottrup H, Lolk A, Wermuth L, Jacobsen S, Laugesen LP, Gergelyffy RG, Høgh P, Bjerregaard E, Andersen BB, Siersma V, Johannsen P, Cotman CW, Waldemar G, Hasselbalch SG (2016) Moderate-tohigh intensity physical exercise in patients with Alzheimer's disease: A randomized controlled trial. J Alzheimers Dis $\mathbf{5 0}$, 443-453.
[65] Villars H, Dupuy C, Perrin A, Vellas B, Nourhashemi F (2015) Impact of a therapeutic educational program on quality of life in Alzheimer's disease: Results of a pilot study. J Alzheimers Dis 43, 167-176.

[66] Maci T, Pira FL, Quattrocchi G, Nuovo SD, Perciavalle V, Zappia M (2012) Physical and cognitive stimulation in Alzheimer Disease. The GAIA Project: A pilot study. Am J Alzheimers Dis Other Demen 27, 107-113. 